\title{
Predictive value of the neutrophil-to-lymphocyte ratio and hemoglobin insystemic lupus erythematosus
}

\author{
HAITAO YU ${ }^{1,2}$, LILI JIANG $^{3}$, LIQIONG YAO $^{1}$, CHAO GAN $^{1}$, XINWEN HAN $^{4}$, RUIQI LIU $^{2}$ and NA SU ${ }^{1}$ \\ ${ }^{1}$ Department of Clinical Laboratory, The First Hospital of Lanzhou University; ${ }^{2}$ Department of Clinical Laboratory, \\ The First Clinical Medical College of Lanzhou University, Lanzhou, Gansu 730000; ${ }^{3}$ School of Material Science \\ and Technology, Lanzhou University of Technology, Lanzhou, Gansu 730050; ${ }^{4}$ Department of Orthopaedics, \\ The First Hospital of Lanzhou University, Lanzhou, Gansu 730000, P.R. China
}

Received December 4, 2017; Accepted June 1, 2018

DOI: $10.3892 /$ etm.2018.6309

\begin{abstract}
The aim of the present study was to evaluate the association of the neutrophil-to-lymphocyte ratio (NLR) and hemoglobin levels with disease activity in patients with systemic lupus erythematosus (SLE) and to explore their clinical significance in predicting SLE. The present study included 212 patients with SLE and 201 healthy controls. All the clinical characteristics were collected from their medical records. The results revealed that the NLR was elevated and the hemoglobin level was markedly decreased in the patients with SLE compared with the healthy controls. NLR was positively correlated with the SLE Disease Activity Index 2000 (SLEDAI-2K), erythrocyte sedimentation rate (ESR) and C-reactive protein (CRP), whereas it was not correlated with C3 or C4. The hemoglobin level was negatively correlated with SLEDAI-2K, ESR and CRP and positively correlated with C3 and C4. In addition, NLR $[\operatorname{EXP}(B)=1.986 ; 95 \%$ confidence interval $(\mathrm{CI})$, $1.432-2.753 ; \mathrm{P}=0.001]$ and hemoglobin $[\operatorname{EXP}(\mathrm{B})=0.947 ; 95 \%$ CI, 0.929-0.965; $\mathrm{P}=0.001]$ were independent predictive factors of SLE. The optimal NLR cut-off value for predicting SLE was 2.075 , with $71.14 \%$ sensitivity and $69.57 \%$ specificity, whereas the optimal hemoglobin cut-off value was $131.5 \mathrm{mg} / \mathrm{l}$, with $75.79 \%$ sensitivity and $77.98 \%$ specificity. In addition, high NLR together with low hemoglobin levels and high NLR or low hemoglobin levels had increased positive predictive values (86.05 and 66.95, respectively). High NLR with low hemoglobin levels and high NLR or low hemoglobin levels also had higher sensitivity (64.91 and 92.40, respectively) and specificity (64.91 and 18.95, respectively), compared with high NLR alone or low hemoglobin alone. In conclusion, NLR and
\end{abstract}

Correspondence to: Mrs. Liqiong Yao, Department of Clinical Laboratory, The First Hospital of Lanzhou University, 1 West Road East Hills, Lanzhou, Gansu 730000, P.R. China

E-mail: yuhaitao7707@163.com

Key words: neutrophil-to-lymphocyte ratio, hemoglobin, predictive value, systemic lupus erythematosus, disease activity hemoglobin may reflect SLE disease activity and may be used as markers for predicting the outcome of SLE.

\section{Introduction}

Systemic lupus erythematosus (SLE) is a chronic inflammatory autoimmune disease that can affect various organ systems. In China, SLE occurs at an incidence of $31-70$ per 100,000 individuals (1). SLE is characterized by high heterogeneity, a complex pathophysiology and various clinical manifestations; thus, no test alone is sufficiently sensitive or specific for diagnosis (2).

Currently, the SLE disease activity index 2000 (SLEDAI-2K) score is widely utilized to assess disease activity in SLE patients $(3,4)$. However, it could be too complex to use in routine clinical practice. Conventional methods, including erythrocyte sedimentation rate (ESR) and C-reactive protein (CRP) are not in complete accordance with the activity of SLE $(5,6)$. Therefore, there is significant interest in the identification of biomarkers that can predict SLE and quantify disease activity, although a single biomarker is unlikely to replace clinical evaluation due to the heterogeneity of this disease (7).

The neutrophil-to-lymphocyte ratio (NLR) and hemoglobin are parts of a routine complete blood count. NLR can be easily calculated using peripheral blood, and it has been identified as a new index of inflammatory biomarkers in a number of diseases (8-10). Hu et al (11) reported that increased NLR was positively correlated with primary Sjögren's syndrome (pSS). Similarly, Sen et al (12) reported that NLR may reflect systemic inflammation in psoriasis patients. Although Qin et al (13) found that NLR may reflect inflammatory responses and disease activity in SLE patients, the predictive value of NLR in connection with hemoglobin levels in SLE has not been determined. The hemoglobin level may be used as an indicator of active inflammatory disease $(14,15)$. Low hemoglobin levels have been found to be significantly associated with disease activity in rheumatoid arthritis (16). However, to the best of our knowledge, the predictive value of the hemoglobin level in SLE remains unclear.

In this study, we analyzed the medical records of 212 SLE patients and 201 healthy individuals to determine the possible association of NLR and hemoglobin level with disease activity and inflammatory responses in SLE patients, in order to 
evaluate the predictive value of NLR and hemoglobin level in SLE.

\section{Patients and methods}

Participants. A total of 212 adult patients with SLE were involved in this retrospective study, according to the diagnostic criteria of SLE developed by the American College of Rheumatology in 1997. All of the patients were originally recruited to the First Hospital of Lanzhou University (Lanzhou, China) between July 2013 and January 2017; their medical records were obtained for retrospective use in the present study. The SLE patients with hematological diseases, blood transfusion, malignancies, other autoimmune diseases, lymphoproliferative disorders, infections or hepatosplenic diseases were excluded. In addition, 201 age- and sex-matched healthy subjects who underwent routine physical examinations in the same hospital during the same period were used as the healthy control group. The study protocol was approved by the Research Ethics Committee of the First Hospital of Lanzhou University (no. LDYYLL201731).

Laboratory values and clinical assessment. Age and sex were extracted from the electronic medical records system database. Blood tests, such as red blood cell count, white blood cell count, neutrophil count, neutrophil ratio in the white blood cell count, lymphocyte count, lymphocyte ratio in the white blood cell count, and hemoglobin, were performed using a hematology analyzer (Sysmex XE-5000; Sysmex Corporation, Kobe, Japan) with its supporting reagents. The NLR was calculated as the absolute neutrophil count divided by the absolute lymphocyte count. Furthermore, CRP, complement component 3 (C3) and $\mathrm{C} 4$ were analyzed using an automatic protein analyzer (Siemens Healthcare Diagnostics, Eschborn, Germany). ESR was measured with an automatic ESR analyzer (ORON-200; HORRON XLH Medical Electronics, Shenzhen, China). In addition, SLEDAI-2K, a global index based on symptoms and laboratory findings, was used to assess SLE disease activity based on the symptoms and laboratory test results (17).

Statistical analysis. The descriptive statistics are presented as mean \pm standard deviation for continuous variables and percentages of the number for categorical variables. The unpaired t-test or Mann-Whitney U-test was used to compare the two independent groups according to distribution state. Pearson's approach was used to quantify the correlation between variables. A receiver operating characteristic (ROC) curve was constructed to determine the predictive value of NLR and hemoglobin in the patient group. A P-value of $<0.05$ was considered to indicate statistically significant differences. All statistical studies were conducted with the SPSS program v.16.0 (SPSS, Inc., Chicago, IL, USA).

\section{Results}

Characteristics of the participants. The demographic characteristics, clinical characteristics and laboratory findings of the 212 SLE patients and 201 healthy controls are listed in Table I. There was no significant difference in age, sex or neutrophil count between the SLE and healthy control groups.
The neutrophil ratio in the white blood cell count was markedly increased, and the red blood cell count, white blood cell count, lymphocyte count and lymphocyte ratio in the white blood cell count were significantly decreased in SLE patients $(\mathrm{P}<0.001$; Table I).

NLR was increased and hemoglobin was decreased in SLE patients compared with healthy controls. As shown in Fig. 1, NLR was significantly elevated $(\mathrm{P}<0.001$; Fig. 1A) and the hemoglobin level was markedly decreased $(\mathrm{P}<0.001$; Fig. 1B) in total SLE patients compared with healthy controls. In order to investigate the association of NLR and hemoglobin levels with SLE patient sex, the cohort was divided into females and males. NLR was significantly elevated in female SLE patients compared with healthy female controls $(\mathrm{P}<0.001)$, whereas there was no statistically significant difference between male SLE patients compared with healthy male controls (Fig. 1C and D). The hemoglobin level was markedly decreased in both female and male patients with SLE (Fig. 1E and F).

Correlations of NLR and hemoglobin with disease activity in SLE. The study demonstrated that NLR was positively correlated with SLEDAI-2K $(r=0.211, P=0.003$; Fig. 2A), ESR ( $r=0.298, P<0.001$; Fig. 2B) and CRP ( $r=0.379, \mathrm{P}<0.001$; Fig. 2C), but not with $\mathrm{C} 3$ ( $\mathrm{r}=-0.054, \mathrm{P}=0.450$; Fig. 2D) or $\mathrm{C} 4$ $(\mathrm{r}=-0.060, \mathrm{P}=0.412$; Fig. $2 \mathrm{E})$. Furthermore, the hemoglobin level was negatively correlated with SLEDAI-2K ( $\mathrm{r}=-0.184$, $\mathrm{P}=0.019$; Fig. 3A), ESR ( $\mathrm{r}=-0.557, \mathrm{P}<0.001$; Fig. 3B) and CRP $(r=-0.157, P<0.007$; Fig. 3C), whereas it was positively correlated with $\mathrm{C} 3(\mathrm{r}=0.303, \mathrm{P}<0.001$; Fig. 3D) and $\mathrm{C} 4(\mathrm{r}=0.293$, $\mathrm{P}=0.003$; Fig. 3E).

NLR and hemoglobin may predict the development of SLE. The risk factors were entered into a logistic regression analysis. The results demonstrated that NLR $[\operatorname{EXP}(B)=1.986 ; 95 \% \mathrm{CI}$, 1.432-2.753; $\mathrm{P}=0.001]$, hemoglobin $[\mathrm{EXP}(\mathrm{B})=0.947 ; 95 \% \mathrm{CI}$, 0.929-0.965; $\mathrm{P}=0.001], \mathrm{C} 3(\mathrm{P}=0.001)$ and $\mathrm{C} 4(\mathrm{P}=0.070)$ were independent predictive factors in SLE patients (Table II).

ROC curve analysis was performed to determine the cut-off value of NLR and hemoglobin to predict SLE, and it revealed that the optimal clinical cut-off level of NLR was 2.075, with a sensitivity of $71.14 \%$ and a specificity of $69.57 \%$. The optimal cut-off level of hemoglobin was $131.5 \mathrm{~g} / \mathrm{l}$, with a sensitivity of $75.79 \%$ and a specificity of $77.98 \%$. There was no statistically significant difference in the area under the ROC curve between NLR and hemoglobin ( $\mathrm{P}>0.05$; Fig. 4 and Table III).

Predictive value of NLR and hemoglobin in SLE. Although all positive likelihood ratios of high NLR and low hemoglobin levels were $<10$ in predicting SLE, simultaneously high NLR and low hemoglobin levels, and high NLR or low hemoglobin levels, had higher positive predictive values (86.05 and 66.95, respectively) and positive likelihood ratios (3.42 and 1.14, respectively) compared with a high NLR level alone or a low hemoglobin level alone (Table IV). Moreover, high NLR with low hemoglobin levels and high NLR or low hemoglobin exhibited higher sensitivity (64.91 and 92.40, respectively) and specificity (64.91 and 18.95, respectively), compared with a high NLR level alone or a low hemoglobin level alone (Table IV). 
Table I. Characteristics of the participants.

\begin{tabular}{|c|c|c|c|c|c|}
\hline \multirow[b]{2}{*}{ Characteristic } & \multicolumn{2}{|c|}{ SLE patients $(n=212)$} & \multicolumn{2}{|c|}{ Healthy controls $(n=201)$} & \multirow[b]{2}{*}{ P-value } \\
\hline & $\mathrm{n}$ & Results & $\mathrm{n}$ & Results & \\
\hline Age (years) & 212 & $40.19 \pm 15.24$ & 201 & $41.45 \pm 12.08$ & 0.351 \\
\hline Sex (male/female) & 212 & $23 / 189$ & 201 & $20 / 181$ & 0.766 \\
\hline $\mathrm{RBC}\left(\mathrm{x} 10^{12} / 1\right)$ & 174 & $3.93 \pm 0.89$ & 201 & $4.66 \pm 0.36$ & $<0.0001^{\mathrm{c}}$ \\
\hline WBC $\left(\times 10^{9} / 1\right)$ & 212 & $5.36 \pm 2.94$ & 201 & $6.04 \pm 1.28$ & $0.002^{\mathrm{b}}$ \\
\hline $\operatorname{Lym}\left(\times 10^{9} / 1\right)$ & 212 & $1.19 \pm 0.70$ & 201 & $0.96 \pm 0.46$ & $<0.0001^{\mathrm{c}}$ \\
\hline Lymphocyte (\%) & 212 & $24.40 \pm 11.09$ & 201 & $32.76 \pm 5.18$ & $<0.0001^{\mathrm{c}}$ \\
\hline $\mathrm{Neu}\left(\mathrm{x} 10^{9} / \mathrm{l}\right)$ & 212 & $3.93 \pm 2.48$ & 201 & $3.65 \pm 0.93$ & $0.048^{\mathrm{a}}$ \\
\hline Neutrophil (\%) & 212 & $66.60 \pm 12.70$ & 201 & $60.07 \pm 5.44$ & $<0.0001^{\mathrm{c}}$ \\
\hline CRP (mg/l) & 189 & $14.59 \pm 2.37$ & 30 & $2.90 \pm 1.04$ & $<0.0001^{\mathrm{c}}$ \\
\hline C3 (g/l) & 201 & $0.70 \pm 0.33$ & 30 & $1.20 \pm 0.27$ & $<0.0001^{\mathrm{c}}$ \\
\hline $\mathrm{C} 4(\mathrm{~g} / \mathrm{l})$ & 194 & $0.14 \pm 0.11$ & 30 & $0.34 \pm 0.08$ & $<0.0001^{\mathrm{c}}$ \\
\hline $\operatorname{ESR}(\mathrm{mm} / \mathrm{h})$ & 192 & $42.08 \pm 3.36$ & - & - & - \\
\hline SLEDAI-2K & 141 & $10.67 \pm 6.63$ & - & - & \\
\hline
\end{tabular}

Data are expressed as the mean \pm standard deviation. ${ }^{\mathrm{a}} \mathrm{P}<0.05,{ }^{\mathrm{b}} \mathrm{P}<0.01$ and ${ }^{\mathrm{c}} \mathrm{P}<0.0001$. $\mathrm{RBC}$, red blood cell count; WBC, white blood cell count; Lym, lymphocyte count; Neu, neutrophil count; CRP, C-reactive protein; ESR, erythrocyte sedimentation rate; SLEDAI-2K, SLE Disease Activity Index 2000.
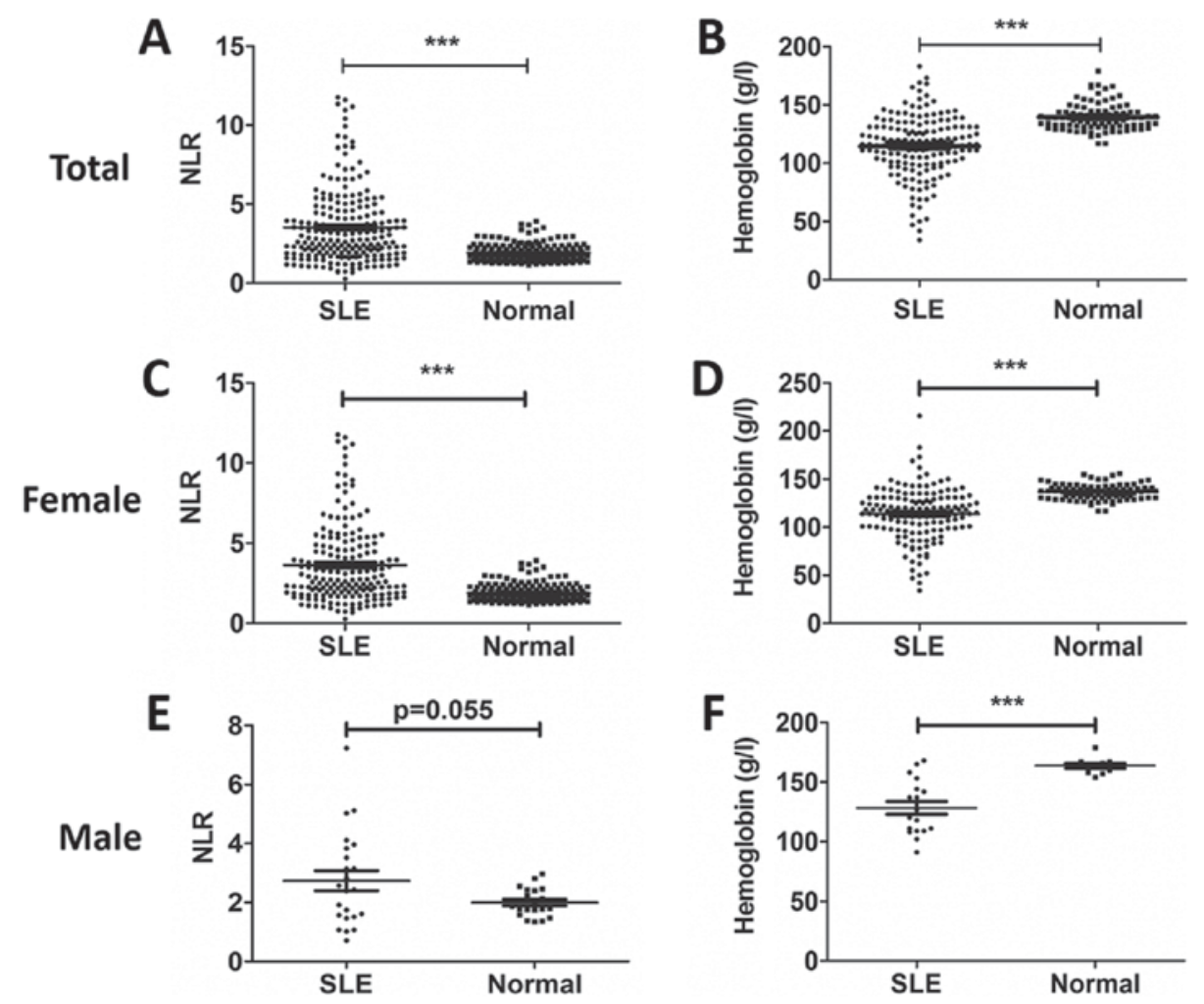

Figure 1. NLR and hemoglobin level in SLE and healthy controls. (A) NLR in total SLE patients and healthy controls, (B) hemoglobin in total SLE patients and healthy controls, (C) NLR in female SLE patients and healthy controls, (D) hemoglobin in female SLE patients and healthy controls, (E) NLR in male SLE patients and healthy controls, (F) hemoglobin in male SLE patients and healthy controls. ${ }^{* * *}$ P $<0.001$, as indicated. NLR, neutrophil-to-lymphocyte ratio; SLE, systemic lupus erythematosus.

\section{Discussion}

This study demonstrated that NLR was increased and hemoglobin was decreased in SLE patients, no matter in female or male. Another key finding was that NLR was positively correlated with SLEDAI-2K, ESR and CRP, while hemoglobin level was negatively correlated with SLEDAI-2K, ESR and CRP, and positively correlated with $\mathrm{C} 3$ and $\mathrm{C} 4$. Furthermore, NLR 

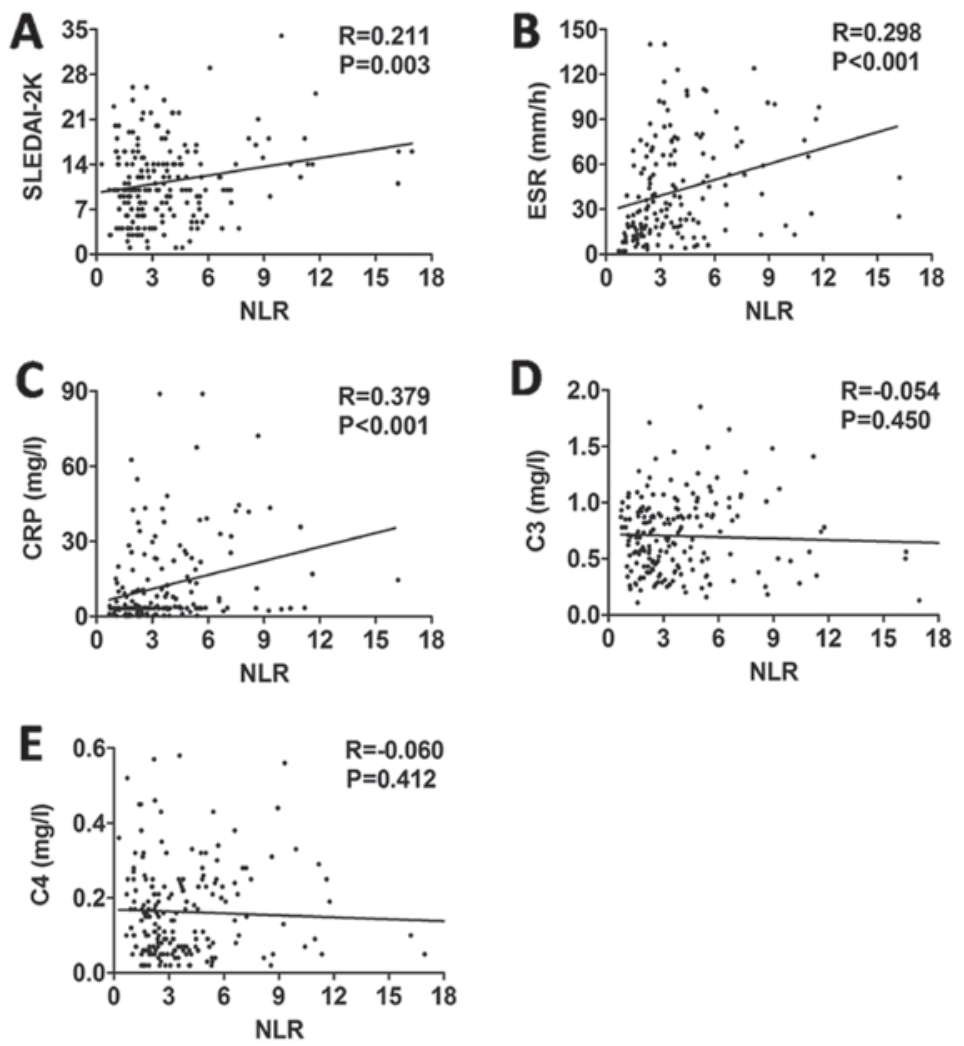

Figure 2. Correlations between NLR and (A) SLEDAI-2K, (B) ESR, (C) CRP, (D) C3, and (E) C4 in SLE patients. Data were analyzed using Pearson's approach. NLR, neutrophil-to-lymphocyte ratio; SLEDAI-2K, SLE Disease Activity Index 2000; ESR, erythrocyte sedimentation rate; CRP, C-reactive protein; SLE, systemic lupus erythematosus.
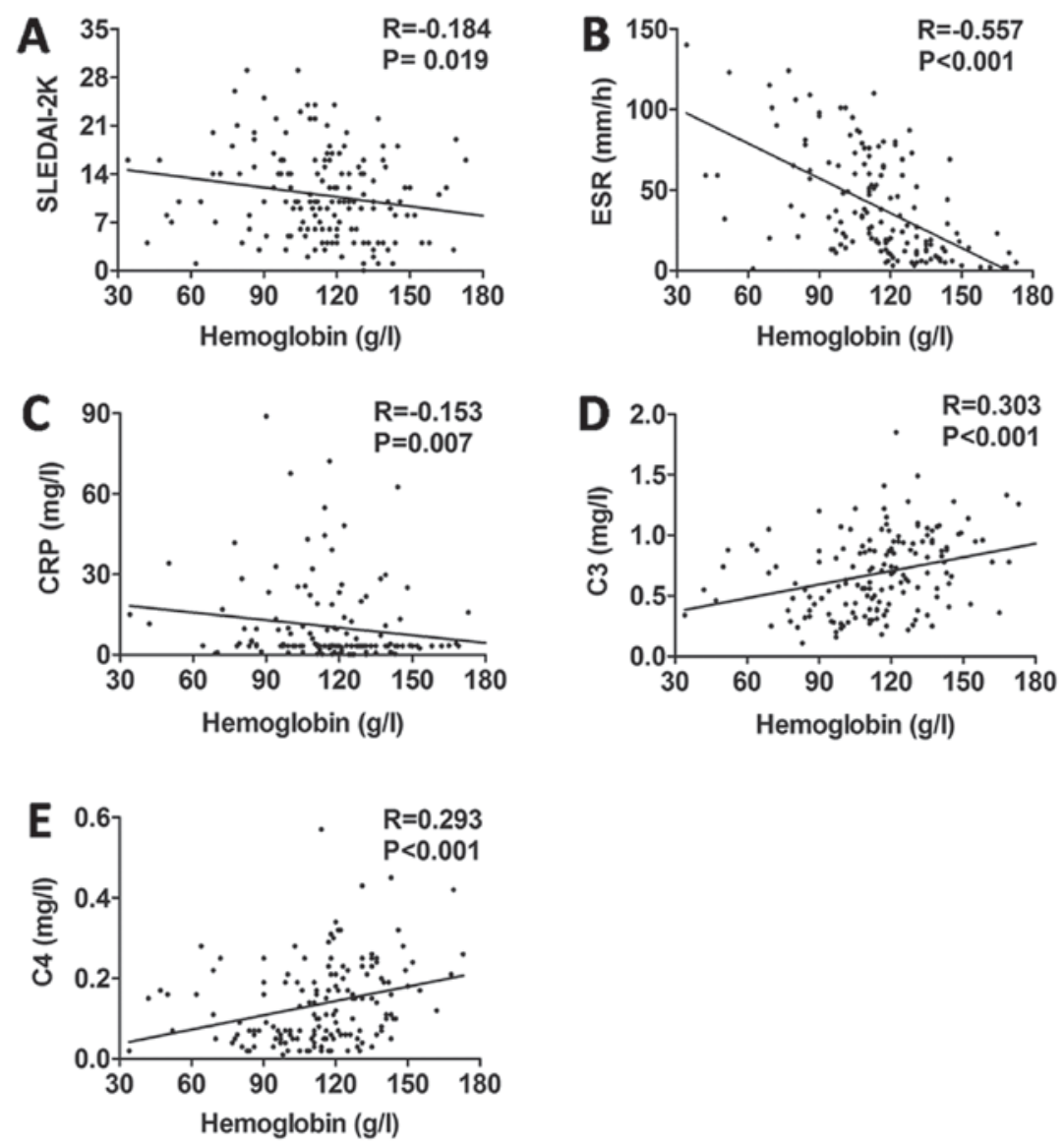

Figure 3. Correlations between hemoglobin and (A) SLEDAI-2K, (B) ESR, (C) CRP, (D) C3 and (E) C4 in SLE patients. Data were analyzed using Pearson's approach. SLEDAI-2K, SLE Disease Activity Index 2000; ESR, erythrocyte sedimentation rate; CRP, C-reactive protein; SLE, systemic lupus erythematosus. 
Table II. Logistic regression analysis of factors independently associated with SLE.

\begin{tabular}{lccc}
\hline Variable & P-value & EXP(B) & 95\% CI of EXP(B) \\
\hline NLR & 0.001 & 1.986 & $1.432-2.753$ \\
Hemoglobin & 0.001 & 0.947 & $0.929-0.965$ \\
C3 & 0.001 & 0.004 & $0.000-0.123$ \\
C4 & 0.007 & 0.049 & $0.005-0.430$ \\
CRP & 0.080 & 1.393 & $0.961-2.017$ \\
\hline
\end{tabular}

SLE, systemic lupus erythematosus; NLR, neutrophil-to-lymphocyte ratio; $\mathrm{CI}$, confidence interval; $\mathrm{CRP}, \mathrm{C}$-reactive protein.

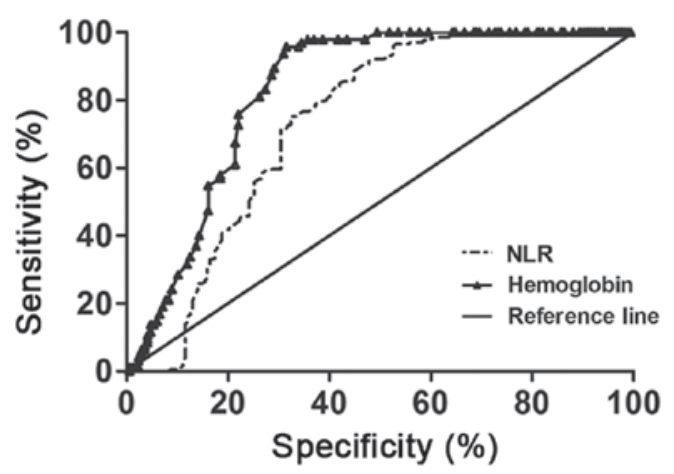

Figure 4. Receiver operating characteristics curve analysis for NLR and hemoglobin. NLR, neutrophil-to-lymphocyte ratio.

and hemoglobin were independent predictive factors for SLE. These findings indicate that NLR and hemoglobin, which are easily detectable and applicable laboratory parameters, maybe predictive factors for SLE and reflect inflammatory response and disease activity in SLE patients.

It is well known that the blood composition undergoes relative changes under conditions of systemic inflammation, typically represented by neutrophilia, lymphopenia and anemia (18). In recent years, neutrophil count, lymphocyte count and hemoglobin levels have been identified as biomarkers of inflammation in several diseases. NLR has been used in combination with other inflammatory markers to quantify systemic inflammation in both auto- and non-autoimmune diseases (13). Accumulating evidence has revealed that NLR is a reliable indicator of inflammation $(11,19,20)$. Qin et al (13) reported that NLR was increased in SLE patients in a study of a cohort of 154 SLE patients and 151 healthy controls. In accordance with the results of previous studies, our results further confirmed that NLR was positively correlated with SLEDAI-2K, a widely utilized indicator to assess disease activity in SLE patients, and ESR and CRP, which are inflammatory indices also used as markers of SLE disease activity. Interestingly, our research also demonstrated that there is no correlation of NLR with C3 and C4, which are biomarkers of SLE disease activity. This inconsistency may be the reason why medical intervention in SLE patients markedly affects $\mathrm{C} 3$ and $\mathrm{C} 4$ (21), or an indication that the abovementioned parameters are involved in separate biological processes in SLE.
Anemia in SLE usually develops in the context of chronic systemic inflammation, and low hemoglobin levels have been found to be an indicator of subclinical inflammatory disease $(14,15)$. However, hemoglobin is not considered to be a readily available acute phase response biomarker, unlike ESR and CRP, which are routinely used to estimate the activity of systemic inflammation. Previous studies reported that the hemoglobin level was increased after starting targeted rheumatoid arthritis treatment $(22,23)$, but failed to report longitudinal changes in composite disease activity indices (23). To the best of our knowledge, there are no studies on the direct association between hemoglobin and SLE. The present study demonstrated that the level of hemoglobin was significantly lower in SLE patients compared with that in healthy controls. In order to further elucidate the association between hemoglobin and SLE, we evaluated the correlations between hemoglobin level and SLEDAI-2K, ESR, CRP, C3 and C4 in patients with SLE, and found that hemoglobin is negatively correlated with SLE disease activity. All these findings suggest that NLR and hemoglobin may be used to reflect the inflammatory response and disease activity in SLE patients.

In this study, logistic regression analysis revealed that SLE wasindependentlyassociated withNRL, hemoglobin,C3 and C4, while NLR exhibited a moderate association $[\operatorname{Exp}(B)=1.986]$, hemoglobin a moderate association $[\operatorname{Exp}(B)=0.947]$, and $C 3$ and $\mathrm{C} 4$ a less significant association $[\operatorname{Exp}(\mathrm{B})=0.004,0.049$, respectively]. Moreover, the area under the ROC curve of NLR and hemoglobin was 0.735 (with a sensitivity of $71.14 \%$ and a specificity of $69.57 \%$ ) and 0.825 (with a sensitivity of $75.79 \%$ and a specificity of $77.98 \%$ ), respectively, in differentiating between SLE patients and normal subjects. Therefore, the two indices NLR and hemoglobin have predictive power for SLE, and the increased NLR and decreased hemoglobin levels observed at the onset of inflammation are significantly associated with the presence of SLE. In addition, the combined results in the present study demonstrated that simultaneously high NLR and low hemoglobin levels, and high NLR or low hemoglobin levels, had higher positive predictive values ( 86.05 and 66.95, respectively), with higher sensitivity (64.91 and 92.40 , respectively) and specificity (84.05 and 18.95 , respectively) in diagnosing SLE compared with high NLR alone or low hemoglobin alone, although all positive likelihood ratios of high NLR and low hemoglobin levels were $<10$. This suggests that NLR and hemoglobin should be measured simultaneously for a more reliable clinical diagnosis in SLE. NLR and hemoglobin measurement is easy, cost-effective and readily available compared with tests for other inflammatory cytokines. Therefore, they are valuable clinical tools that may serve as biomarkers for inflammatory response and for predicting SLE. Due to the complexity of NLR and hemoglobin, they are not included in major management recommendations for SLE at present, as further clinical decision-making is required.

There were certain limitations to the present study. First, this was a single-center retrospective study, and additional larger, multicenter studies are needed to verify our results. Furthermore, the exact mechanism underlying the involvement of NLR and hemoglobin in SLE was not investigated. Finally, the participants were only Chinese, and the inclusion of other ethnic groups is required in further investigations. 
Table III. Analysis of the area under the ROC curve for NLR and hemoglobin.

\begin{tabular}{lccccc}
\hline Test result variable & Area & $\begin{array}{c}\text { Standard } \\
\text { error }^{\mathrm{a}}\end{array}$ & $\begin{array}{c}\text { Asymptotic } \\
\text { significance }^{\mathrm{b}}\end{array}$ & & \multicolumn{2}{c}{ Asymptotic 95\% confidence interval } \\
\cline { 6 - 6 } NLR & 0.735 & 0.026 & 0.000 & Lower bound & Upper bound \\
Hemoglobin levels & 0.825 & 0.025 & 0.000 & 0.684 & 0.786 \\
\hline
\end{tabular}

${ }^{a}$ Under the non-parametric assumption. ${ }^{b} \mathrm{Null}$ hypothesis: True area $=0.5$. The test result variables have at least one tie between the positive actual state group and the negative actual state group. Statistics may be biased. ROC, receiver operating characteristics; NLR, neutrophil-to-lymphocyte ratio.

Table IV. Statistical evaluation of NLR and hemoglobin levels in predicting SLE.

\begin{tabular}{|c|c|c|c|c|c|c|c|}
\hline Characteristic & $\begin{array}{c}\text { Sensitivity } \\
(\%)\end{array}$ & $\begin{array}{l}\text { Specificity } \\
(\%)\end{array}$ & $\begin{array}{l}\text { Accuracy } \\
(\%)\end{array}$ & $\begin{array}{l}\text { Positive predicted } \\
\text { value }(\%)\end{array}$ & $\begin{array}{c}\text { Negative predicted } \\
\text { value }(\%)\end{array}$ & $\begin{array}{c}\text { Positive } \\
\text { likelihood ratio }\end{array}$ & $\begin{array}{c}\text { Negative } \\
\text { likelihood ratio }\end{array}$ \\
\hline NLR up alone & 6.43 & 78.95 & 32.33 & 35.48 & 31.91 & 0.31 & 1.19 \\
\hline $\begin{array}{l}\text { Hemoglobin } \\
\text { down alone }\end{array}$ & 22.81 & 61.05 & 36.47 & 51.32 & 30.53 & 0.59 & 1.26 \\
\hline $\begin{array}{l}\text { NLR up and } \\
\text { hemoglobin down }\end{array}$ & 64.91 & 81.05 & 70.68 & 86.05 & 56.2 & 3.42 & 0.43 \\
\hline $\begin{array}{l}\text { NLR up or } \\
\text { hemoglobin down }\end{array}$ & 92.40 & 18.95 & 66.17 & 66.95 & 58.06 & 1.14 & 0.4 \\
\hline
\end{tabular}

NLR, neutrophil-to-lymphocyte ratio; SLE, systemic lupus erythematosus.

In conclusion, the present study has demonstrated that increased NLR and decreased hemoglobin levels were found in SLE patients, and that they were correlated with inflammatory response and disease activity. These findings suggest that NLR and hemoglobin may be useful markers in predicting SLE and assessing disease activity. However, further studies are required to elucidate the exact role of NLR and hemoglobin in SLE.

\section{Acknowledgements}

Not applicable.

\section{Funding}

This study was supported by the Health Industry Research Program Management of Gansu Province (grant no. GWGL 2014-48) and the First Hospital of Lanzhou University Foundation (grant no. ldyyyn2017-19).

\section{Availability of data and materials}

The datasets used and/or analyzed during the current study are available from the corresponding author on reasonable request.

\section{Authors' contributions}

HY conceived and designed the present study, performed analysis and acquired funding. LY and XH collected the data. RL, CG and NS performed the experiments. HY drafted the manuscript. LJ designed and revised the manuscript. HY, LJ, LY, CG, XH, RL and NS gave final approval of the version to be published.

\section{Ethics approval and consent to participate}

The study protocol was approved by the Research Ethics Committee of the First Hospital of Lanzhou University (no. LDYYLL201731).

\section{Patient consent for publication}

Not applicable.

\section{Competing interests}

The authors declare that they have no competing interests.

\section{References}

1. Zeng QY, Chen R, Darmawan J, Xiao ZY, Chen SB, Wigley R, Le Chen S and Zhang NZ: Rheumatic diseases in China. Arthritis Res Ther 10: R17, 2008.

2. Liu CC and Ahearn JM: The search for lupus biomarkers. Best Pract Res Clin Rheumatol 23: 507-523, 2009.

3. Yuan J, Li LI, Wang Z, Song W and Zhang Z: Dyslipidemia in patients with systemic lupus erythematosus: Association with disease activity and B-type natriuretic peptide levels. Biomed Rep 4: 68-72, 2016.

4. Urowitz MB, Isenberg DA and Wallace DJ: Safety and efficacy of hCDR1 (Edratide) in patients with active systemic lupus erythematosus: Results of phase II study. Lupus Sci Med 2: e000104, 2015. 
5. Osei-Bimpong A, Meek JH and Lewis SM: ESR or CRP? A comparison of their clinical utility. Hematology 12: 353-357, 2007.

6. Gaitonde S, Samols D and Kushner I: C-reactive protein and systemic lupus erythematosus. Arthritis Rheum 59: 1814-1820, 2008

7. Petri M: Disease activity assessment in SLE: Do we have the right instruments? Ann Rheum Dis 66 (Suppl 3): iii61-iii64, 2007.

8. Afari ME and Bhat T: Neutrophil to lymphocyte ratio (NLR) and cardiovascular diseases: An update. Expert Rev Cardiovasc Ther 14: 573-577, 2016.

9. Balta S, Celik T, Mikhailidis DP, Ozturk C, Demirkol S, Aparci $\mathrm{M}$ and Iyisoy A: The relation between atherosclerosis and the neutrophil-lymphocyte ratio. Clin Appl Thromb Hemost 22: 405-411, 2016.

10. Kim DS, Shin D, Lee MS, Kim HJ, Kim DY, Kim SM and Lee MG: Assessments of neutrophil to lymphocyte ratio and platelet to lymphocyte ratio in Korean patients with psoriasis vulgaris and psoriatic arthritis. J Dermatol 43: 305-310, 2016.

11. Hu ZD, Sun Y, Guo J, Huang YL, Qin BD, Gao Q, Qin Q, Deng AM and Zhong RQ: Red blood cell distribution width and neutrophil/lymphocyte ratio are positively correlated with disease activity in primary Sjögren's syndrome. Clin Biochem 47: 287-290, 2014.

12. Sen BB, Rifaioglu EN, Ekiz O, Inan MU, Sen T and Sen N: Neutrophil to lymphocyte ratio as a measure of systemic inflammation in psoriasis. Cutan Ocul Toxicol 33: 223-227, 2014

13. Qin B, Ma N, Tang Q, Wei T, Yang M, Fu H, Hu Z, Liang Y, Yang Z and Zhong R: Neutrophil to lymphocyte ratio (NLR) and platelet to lymphocyte ratio (PLR) were useful markers in assessment of inflammatory response and disease activity in SLE patients. Mod Rheumatol 26: 372-376, 2016.

14. Moller B, Scherer A, Förger F, Villiger PM and Finckh A; Swiss Clinical Quality Management Program for Rheumatic Diseases: Anaemia may add information to standardised disease activity assessment to predict radiographic damage in rheumatoid arthritis: A prospective cohort study. Ann Rheum Dis 73: 691-696, 2014.
15. van Steenbergen HW, van Nies JA and van der Helm-van Mil AH: Anaemia to predict radiographic progression in rheumatoid arthritis. Ann Rheum Dis 72: e16, 2013.

16. Smyrnova G: The relationship between hemoglobin level and disease activity in patients with rheumatoid arthritis. Rev Bras Reumatol 54: 437-440, 2014 (In Portuguese).

17. Gladman DD, Ibañez D and Urowitz MB: Systemic lupus erythematosus disease activity index 2000. J Rheumatol 29: 288-291, 2002.

18. Zahorec R: Ratio of neutrophil to lymphocyte count-rapid and simple parameter of systemic inflammation in critically ill. Bratisl Lek Listy 102: 5-14, 2001 (In English, Slovak).

19. Ahsen A, Ulu MS, Yuksel S, Demir K, Uysal M, Erdogan M and Acarturk G: As a new inflammatory marker for familial Mediterranean fever: Neutrophil-to-lymphocyte ratio. Inflammation 36: 1357-1362, 2013.

20. Wu Y, Chen Y, Yang X, Chen L and Yang Y: Neutrophil-tolymphocyte ratio (NLR) and platelet-to-lymphocyte ratio (PLR) were associated with disease activity in patients with systemic lupus erythematosus. Int Immunopharmacol 36: 94-99, 2016.

21. Stohl W, Hiepe F, Latinis KM, Thomas M, Scheinberg MA, Clarke A, Aranow C, Wellborne FR, Abud-Mendoza C, Hough DR, et al: Belimumab reduces autoantibodies, normalizes low complement levels, and reduces select B cell populations in patients with systemic lupus erythematosus. Arthritis Rheum 64: 2328-2337, 2012.

22. Hashimoto M, Fujii T, Hamaguchi M, Furu M, Ito H, Terao C, Yamamoto K, Yamamoto W, Matsuo T, Mori M, et al: Increase of hemoglobin levels by anti-IL-6 receptor antibody (tocilizumab) in rheumatoid arthritis. PLoS One 9: e98202, 2014.

23. Isaacs JD, Harari O, Kobold U, Lee JS and Bernasconi C: Effect of tocilizumab on haematological markers implicates interleukin- 6 signalling in the anaemia of rheumatoid arthritis. Arthritis Res Ther 15: R204, 2013. 